\title{
Cerebrovascular adaptations to cocaine-induced transient ischemic attacks in the rodent brain
}

\author{
Jiang You, ${ }^{1}$ Nora D. Volkow, ${ }^{2}$ Kicheon Park, ${ }^{1}$ Qiujia Zhang, ${ }^{1}$ Kevin Clare, ${ }^{1}$ Congwu Du, ${ }^{1}$ \\ and Yingtian Pan ${ }^{1}$ \\ 'Department of Biomedical Engineering, Stony Brook University, Stony Brook, New York, USA. ${ }^{2}$ National Institute on \\ Alcohol Abuse and Alcoholism, National Institutes of Health, Bethesda, Maryland, USA.
}

\begin{abstract}
Occurrence of transient ischemic attacks (TIA) and cerebral strokes is a recognized risk associated with cocaine abuse. Here, we use a rodent model along with optical imaging to study cocaineinduced TIA and the associated dynamic changes in cerebral blood flow velocity (CBFv) and cerebrovasculature. We show that chronic cocaine exposure in mice resulted in marked cortical hypoperfusion, in significant arterial and venous vasoconstriction, and in a sensitized vascular response to an acute cocaine injection. Starting after 10 days of exposure, an acute cocaine challenge to these mice resulted in a TIA, which presented as hemiparalysis and was associated with an abrupt exacerbation of CBFv. The severity of the TIA correlated with the decreases in cortical CBFv such that the greater the decreases in flow, the longer the TIA duration. The severity of TIA peaked around 17-22 days of cocaine exposure and decreased thereafter in parallel to a reorganization of CBFv from superficial to deep cortical layers, along with an increase in vessel density into these layers. Here, we document for the first time to our knowledge evidence of a TIA in an animal model of chronic cocaine exposure that was associated with profound decreases in CBFv, and we revealed that while the severity of the TIA initially increased with repeated exposures, it subsequently improved in parallel to an increase in the vessel density. This suggests that strategies to accelerate cerebrovascular recovery might be therapeutically beneficial in cocaine abusers.
\end{abstract}

Conflict of interest: The authors have declared that no conflict of interest exists.

Submitted: September 26, 2016 Accepted: January 24, 2017 Published: March 9, 2017

\section{Reference information:} JCI Insight. 2017;2(5):e90809. https://doi.org/10.1172/jci. insight. 90809.

\section{Introduction}

Cocaine effects on the vascular system significantly contribute to the morbidity and mortality associated with its abuse (1-3). In the brain, the cerebrovascular effects of cocaine are associated with transient ischemic attacks (TIA) - a transient episode of neurological dysfunction without acute infarction - as well as full blown strokes (2). Clinically, TIA is regarded as a portent of risk for subsequent ischemic stroke. In cocaine abusers, TIAs are likely to reflect cocaine's vasoconstricting effects (4). Indeed, cocaine-induced vasospasm has been associated with sudden ischemia (5), and we recently showed that, in rodents, repeated cocaine exposure leads to microischemia and microvascular dysfunction (6). Additionally, platelet aggregation (7) and accelerated atherosclerosis (8) contribute to vascular abnormalities in cocaine abusers. However, as of now, there have not been any studies on cocaine-induced TIA in animal models; hence, information regarding the temporal course of cocaine-induced TIA and its recovery is not available (9) Here, we investigated the temporal course of cocaine-induced TIA in mice, taking advantage of newly developed optical imaging tools.

Specifically, recent advances in optical imaging techniques (e.g., ultrahigh-resolution optical coherence angiography $[\mu \mathrm{OCA}]$ and Doppler tomography $[\mu \mathrm{ODT}])$ have demonstrated their value for studying ischemic injury and neurovascular dysfunction (10), revascularization after traumatic brain injury (TBI) (11), capillary hypoperfusion after arterial occlusion (12), and microischemia after repeated cocaine exposures (6). Here, we used $\mu \mathrm{ODT} / \mu \mathrm{OCA}$ to study cocaine-associated microvascular hypoperfusion, vasoconstriction, and angiogenesis. We employed a chronic cranial window for longitudinal imaging of cerebral blood flow velocity $(\mathrm{CBFv})$ in the mouse's sensorimotor cortex and in parallel monitored behavior (motor function) both during chronic cocaine exposure $(35 \mathrm{mg} / \mathrm{kg} /$ day, i.p.) and after cocaine withdrawal. We documented that mice exposed chronically to cocaine showed vasoconstriction and hypoperfusion, which were 
Table 1. Experimental paradigms for behavioral recording

\begin{tabular}{lccccc}
\hline & Dual windows & & \multicolumn{2}{c}{ Single chronic window } \\
w/ Behavior recording & 7 (Ctrl) & & 7 (CC) & 5 (Ctrl) & 5 (CC) \\
w/o Behavior recording & & 0 & & & \\
& & & & \\
\hline
\end{tabular}

abruptly exacerbated following an acute cocaine challenge that manifested as a TIA episode characterized by hemiparalysis. We also show evidence of vascular adaptions following a TIA that resulted in angiogenesis and cortical CBFv reorganization.

\section{Results}

Behavioral recording of TIA elicited by chronic cocaine. The top panels in Figure 1 compare snapshots of behavioral videos captured from the side-view and top-view cameras of control (Figure 1A, upper 2 rows) and chronic cocaine mice (Figure 1A, lower 2 rows). Abnormal motor behaviors were observed in the chronic cocaine mice following an acute cocaine challenge. Specifically, after a short latency (3'17' $\pm 35 ", n=7$ ) of increased locomotor activity following cocaine injection, all of the chronically cocaine exposed mice $(n=7)$ exhibited disrupted motor behaviors that appeared as the onset of a TIA, which we describe as Phase 1 TIA. Phase 1 TIA was characterized by slow and unsteady gait due to weak and clumsy hind limbs but no obvious hemiparalysis (Figure 1A, Phase 1 TIA) and lasted 2'17" \pm 46 ". Some mice developed more severe locomotor disruption, which we describe as Phase 2 TIA, with symptoms of hemiparalysis (Figure 1A, Phase 2 TIA). Phase 2 TIA was characterized by unilateral paralysis of the front and hind limbs that jeopardized the animal's forward movements and lasted 5'11" \pm 2 '19". During Phase 2 TIA, mice lost balance and either kept turning in unilateral circles contralateral to the paralyzed limbs or laid on the floor (hemiparalysis). As the effects of acute cocaine subsided, the mice gradually recovered, which we describe as Phase 3 TIA, regaining body balance but still showing stiff and staggering locomotion that lasted $8^{\prime} 21^{\prime \prime} \pm 44^{\prime} 39^{\prime \prime}$, before limb function was fully restored. In contrast, the control animals after a saline challenge maintained body balance and normal locomotion during the entire 33-minute monitoring period (see details in Supplemental Movie 1; supplemental material available online with this article; https://doi.org/10.1172/jci.insight.90809DS1).

Figure 1B plots the averages for the prevalence and duration of TIA behavioral episodes in response to acute cocaine as a function of the days of cocaine exposure. The TIA severity index (Figure 1C) expressed as the rate of TIA and the duration of Phases 1,2, and 3, started to increase after day 8 , reached its extremes near days 17-24, but gradually declined thereafter. The decline in TIA's severity with time suggests that there was some degree of vascular recovery or of tolerance to cocaine's vascular effects with chronic administration.

Hypoperfused sensorimotor cortex contralateral to TIA hemiparalysis. The analysis of locomotor behavior suggested that hemiparalysis was an eminent behavioral pattern characteristic of TIA, which led us to hypothesize that it would be associated with cerebrovascular dysfunction in the contralateral sensorimotor cortex. To test this hypothesis, we opened 2 cranial windows over the sensorimotor cortices of each hemisphere on mice (on 7 control and 7 cocaine mice, we opened only one chronic cranial window on the left sensorimotor cortex; see Table 1) who had exhibited locomotor signs of TIA onset the day before so that we could perform $\mu \mathrm{ODT} / \mu \mathrm{OCA}$ scans. Unlike the bilateral CBFv networks of a control mouse, which were high and comparable (Figure 2A, upper panels), those in a TIA mouse (Figure 2B, upper panels) were drastically reduced, leading to bilateral cortical hypoperfusion and unbalanced lateralized perfusion. For example, for a mouse showing right hemiparalysis (Figure 2B, lower panels) the CBFv disruption and ischemia on the contralateral (left) sensorimotor cortex was significantly $(P<0.001$, Figure $3 \mathrm{~A}$, lower left) larger than that in the ipsilateral cortex (right) (Figure 3A, lower right). Moreover, there was a strong correlation ( $\mathrm{r}=1, n=7)$ between hemiparalysis and CBFv dysfunction (ischemia) in the contralateral sensorimotor cortex. Specifically, among the 7 chronic cocaine mice in whom we recorded motor behavior and performed $\mu \mathrm{ODT}$ scans of $\mathrm{CBFv}$ in left and right sensorimotor cortices, 5 showed right hemiparalysis and lower $\mathrm{CBFv}$ in left sensorimotor cortex and 2 showed left hemiparalysis and 
A

응
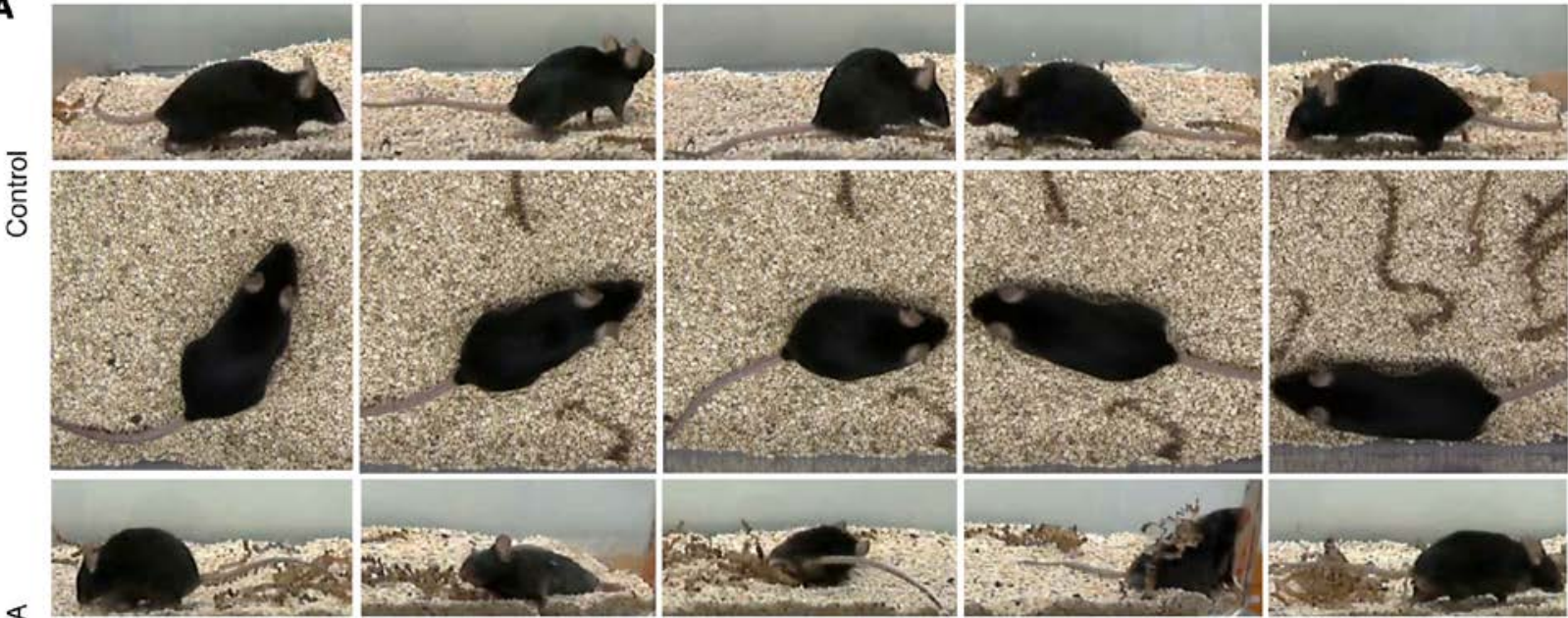

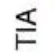
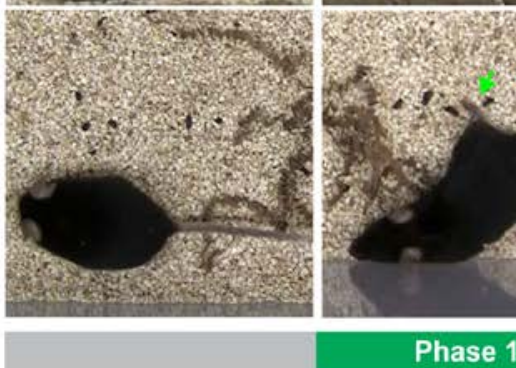

Phase 1

$3{ }^{\prime} 20 "$
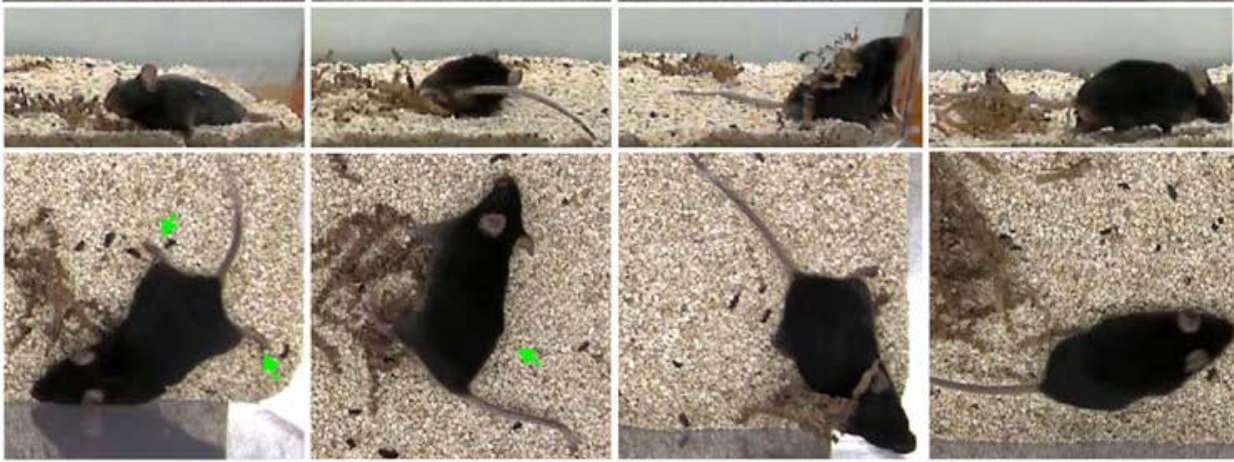

0' Baseline

4

${ }^{*}$ cc $35 \mathrm{mg} / \mathrm{kg} /$ i.p.

B
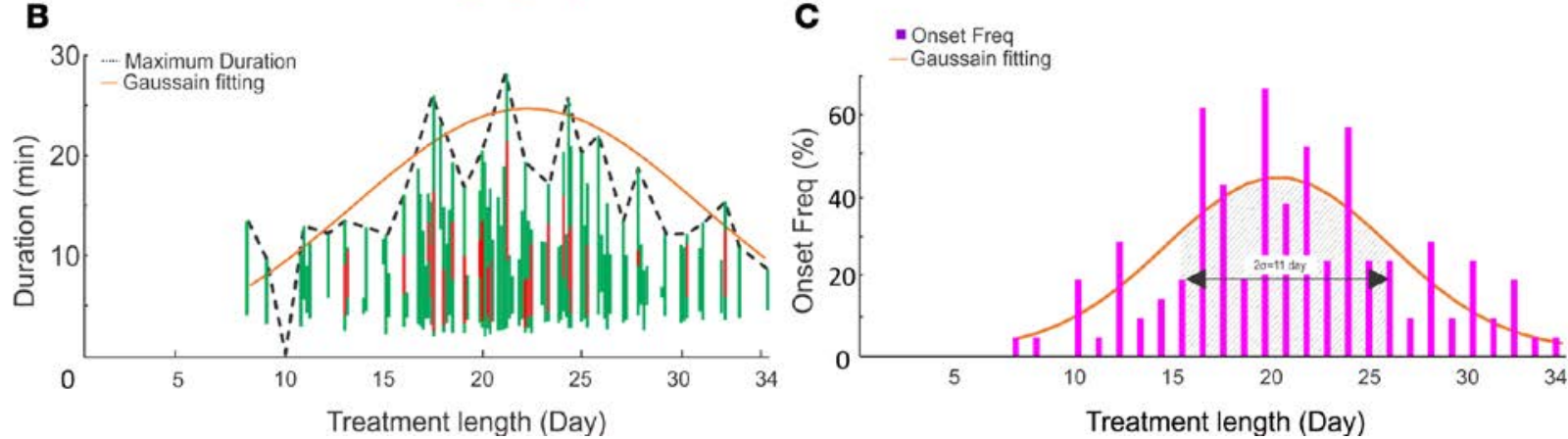

Figure 1. Behavior recording of difference TIA phases and its histogram distribution as a function of time. (A) Upper 2 rows: time-lapse video (side view and en-face view) snapshots of a naive mouse after saline injection ( 0.2 cubic centimeter, i.p.). Lower 2 rows: time-lapse video snapshots of a chronic cocaine (TIA) mouse (day 18) showing TIA after an acute cocaine injection ( $35 \mathrm{mg} / \mathrm{kg}$, i.p.). TIA phases 1,3 , and 2 are highlighted by green and red bars. (B) TIA incidence rates and Phase 1, 2, and 3 duration as a function of days of chronic cocaine treatment $(n=7)$. (C) TIA severity index compounded by the TIA incidence rate and total TIA duration.

lower $\mathrm{CBFv}$ in right sensorimotor cortex. Thus, hemiparalysed limb locomotion was associated with a more severe hypoperfusion of the contralateral than the ipsilateral sensorimotor cortex, consistent with the notion that the motor disruption reflected a TIA. Interestingly, the left sensorimotor cortex appeared to be more vulnerable to chronic cocaine elicited TIA $(n=5)$ than the right $(n=2)(P=0.008)$.

Figure 2C summarizes the longitudinal assessments of TIA episodes and vascular $\mathrm{CBFv}$ changes $(\triangle \mathrm{CBF})$. It shows that TIAs started to emerge approximately after 11 days of chronic cocaine exposure, at which point $\mathrm{CBFv}$ in arteries and veins was about $12.38 \% \pm 6.88 \%$ and $9.17 \% \pm 12.92 \%$ lower than baseline, respectively. Continued repeated exposure led to progressive hypoperfusion in arteries and veins in parallel to an exacerbation of the TIA. Figure 2D shows the correlation between TIA duration and CBFv decreases (compared with baseline), revealing that the greater the flow decrease, the longer the duration of the TIA $(\mathrm{r}=0.733, P<0.0001)$. 
A

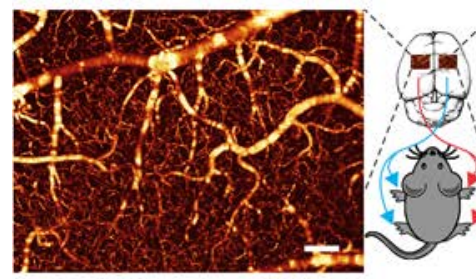

Control
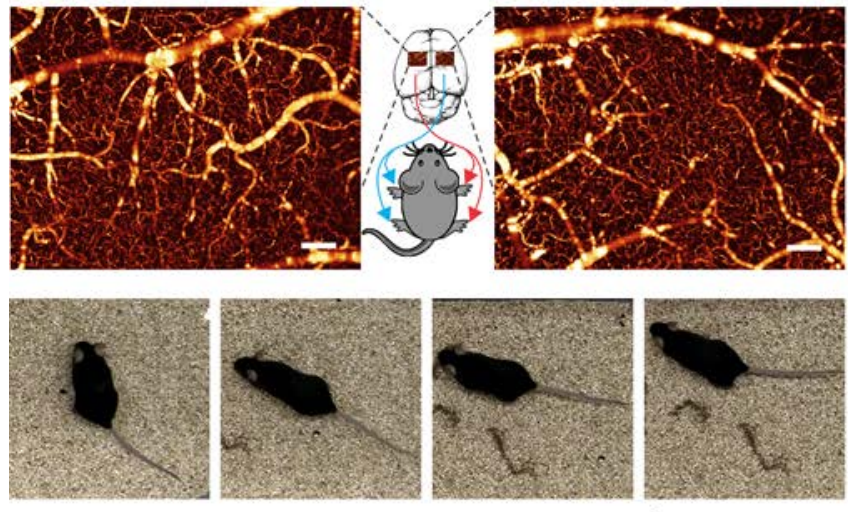

C

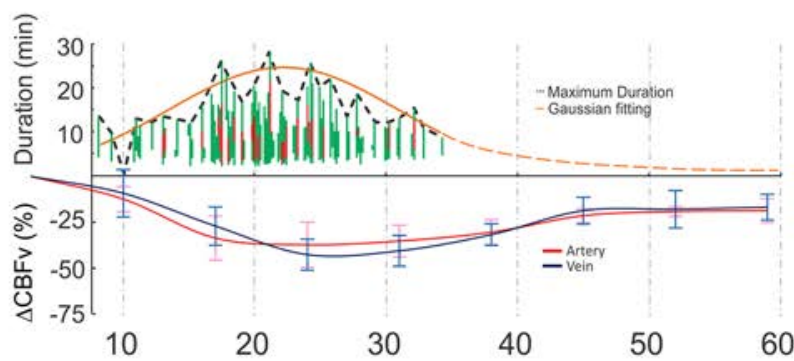

Treatment length (Day)
B

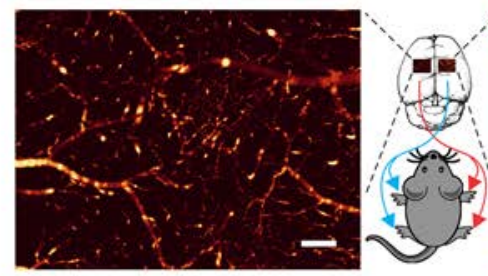

TIA

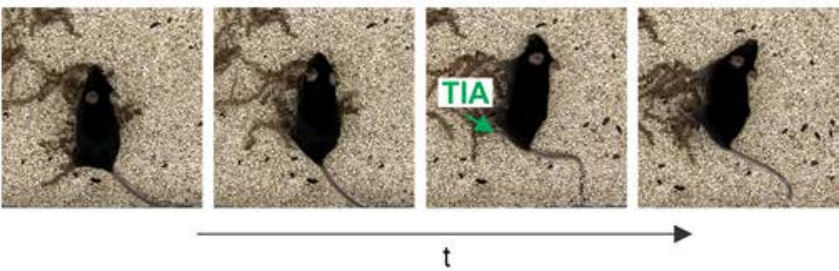

D

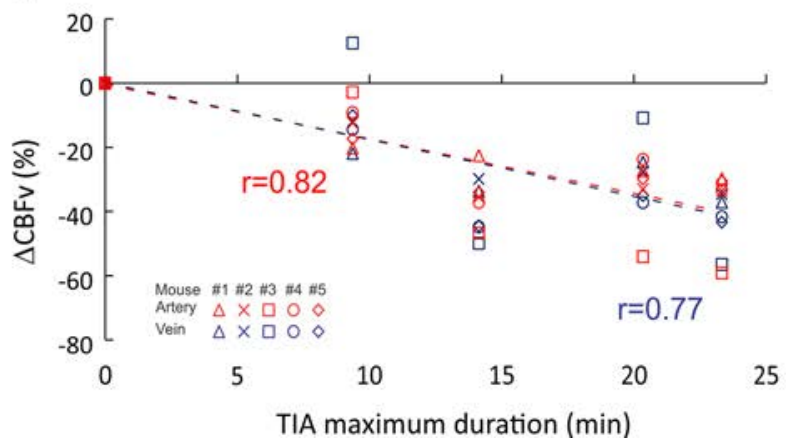

Figure 2. Representative $\mu$ ODT images of the sensorimotor cortex with behavioral images between a control mouse and a TIA mouse after chronic cocaine exposures, and temporal relationship between the TIA incidences and the cortical CBF changes of animals. (A) $\mu$ ODT images of left and right sensorimotor cortices of a control mouse and behavior recording of normal limb locomotion after saline injection. (B) $\mu$ ODT images of left and right sensorimotor cortices of chronic cocaine mouse and behavior recording of hemiparalysed limb after acute cocaine (35 mg/kg, i.p.). Scale bars: $200 \mu \mathrm{m}$. (C) Time courses of TIA incidences $(n=7)$ and the CBFv decreases in arteries and veins of the brains $(n=5)$, indicating that $\Delta$ CBFv decreases precede the TIA. (D) Correlation analysis between $\triangle \mathrm{CBFv}(\%)$ and the maximal duration of TIAs, indicating that TIA were associated with the CBF decrease in the cortical vessels ( $r=0.82$ for arteries and 0.77 for veins, tested by linear regression).

Imbalanced flow deficit. Figure 3 shows quantitative comparisons of CBFv between the left and the right sensorimotor cortex of a control (upper panels) and of a TIA mouse (lower panels). Figure 3, B and $\mathrm{C}$, shows the comparisons of the absolute flow rates in arterial, venous, and capillary compartments between the TIA side and non-TIA side of the sensorimotor cortices of TIA mice $(n=7)$.

Overall, $\mathrm{CBFv}$ in sensorimotor cortices were diminished in a TIA mouse compared with a control mouse and were lower in the contralateral side to the paralysis than in the ipsilateral side (Figure 3A). Specifically, capillary $\mathrm{CBFv}$ was $0.23 \pm 0.03 \mathrm{~mm} / \mathrm{s}$ in somatosensory cortex of control mice $(n=7)$, whereas it was $0.08 \pm 0.05 \mathrm{~mm} / \mathrm{s}$ on the contralateral side $(67.67 \% \pm 19.01 \%$ lower; $P<0.001)$ and 0.17 $\pm 0.05 \mathrm{~mm} / \mathrm{s}$ on the ipsilateral side $(30.15 \% \pm 15.30 \%$ lower; $P=0.014)$ of the TIA side $(n=7)$.

Venous flow was also markedly decreased in mice suffering from a TIA when compared with control mice $(9.45 \pm 0.85 \mathrm{~mm} / \mathrm{s}$ ), corresponding to $2.57 \pm 1.75 \mathrm{~mm} / \mathrm{s}$ on the TIA side (decline of $73.96 \% \pm 16.27 \%$; $P<0.001$ ) and $5.98 \pm 2.66 \mathrm{~mm} / \mathrm{s}$ on the non-TIA side (decline of $38.15 \% \pm 23.44 \% ; P=0.011$ ). Similarly, arterial flow was significantly decreased from $10.99 \pm 1.45 \mathrm{~mm} / \mathrm{s}$ in controls to $6.74 \pm 2.12 \mathrm{~mm} / \mathrm{s}$ on the TIA side (decline of $39.88 \% \pm 13.86 \%, P<0.001$ ), though the non-TIA side did not differ from control $(10.71 \pm 1.85 \mathrm{~mm} / \mathrm{s} ; P=0.620)$.

In addition to hypoperfusion in both sensorimotor cortices, a severe flow disparity was observed between the TIA and non-TIA sides. For instance, the arterial, venous, and capillary flows on the TIA side were $38.25 \% \pm 12.30 \%(P=0.011), 61.39 \% \pm 16.27 \%(P=0.015)$, and $56.87 \% \pm 18.57 \%(P=$ $0.010)$ lower than on the non-TIA side, which is consistent with the findings of a predominant presentation of hemiparalysis rather than of complete paralysis (Figure 1A, TIA Phase 1). 
A

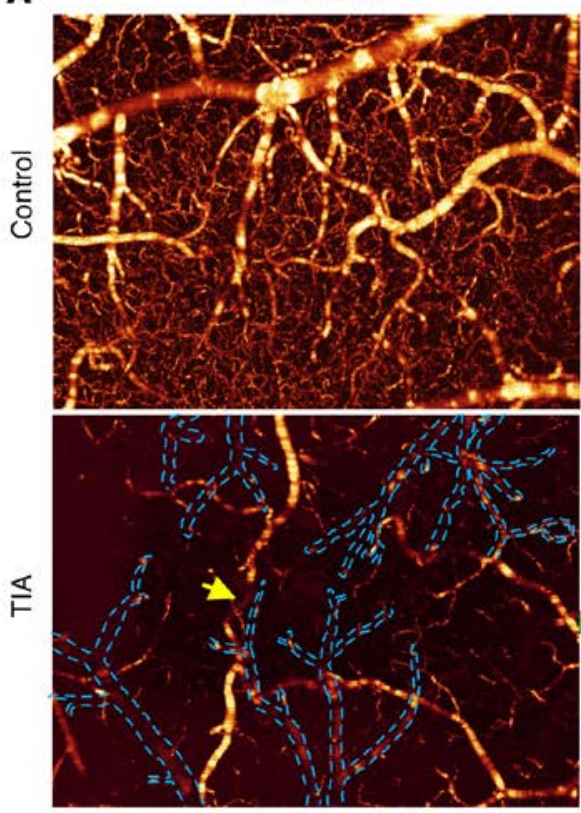

Right cortex

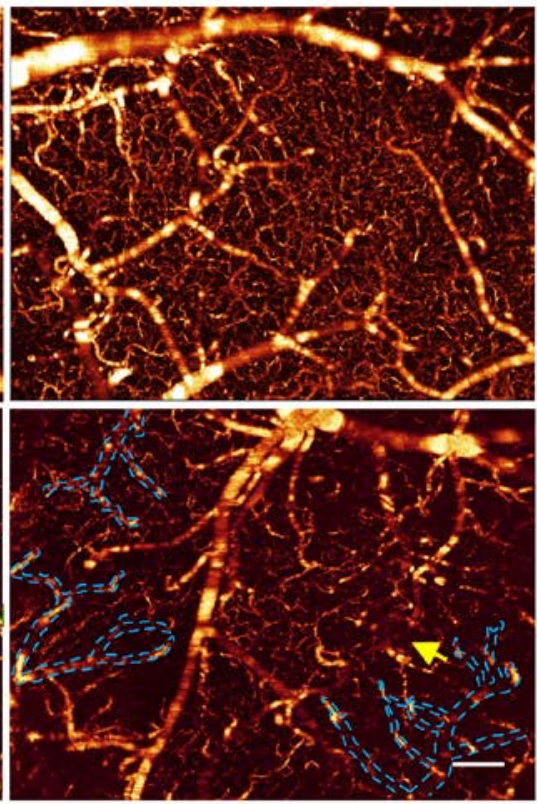

Figure 3. Disparity of cocaine elicited CBFv decreases between left and right sensorimotor cortex by 3D $\mu$ ODT. (A) CBFv network of mouse sensorimotor cortex imaged by $3 D$ $\mu \mathrm{ODT}$ (upper row: control mouse; lower row: TIA mouse). TIA in left sensorimotor cortex correlated with the right limb hemiparalysis. Yellow arrows point to suspected arterial stenosis, and dashed blue lines outline undetectable venous flows. Scale bar: $200 \mu \mathrm{m}$. (B and C) Statistic figures of CBFv rates in arterial, venous, and capillary compartments of left (TIA) and right (non-TIA) sides, respectively. Unpaired 2-tailed Student's $t$ tests or rank sum test were performed for statistical analysis, and average values are shown as the mean $\pm \mathrm{SD}$ in B and C. $n=7$ for each group. ${ }^{*} P<0.05$ ${ }^{* * *} P<0.001$.

\section{B}

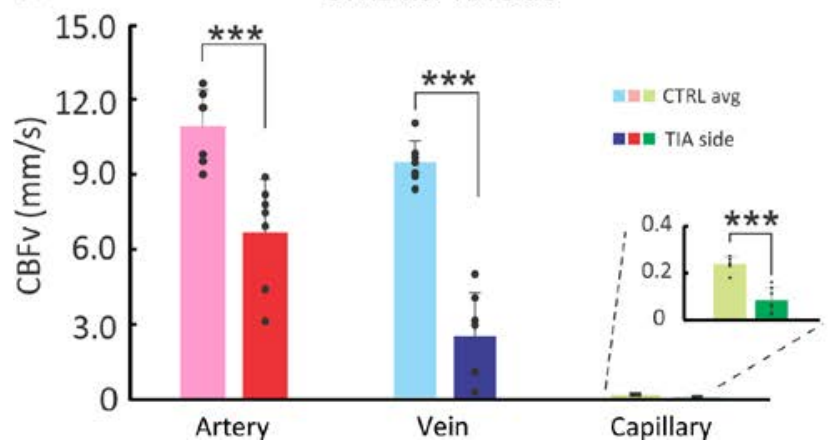

C

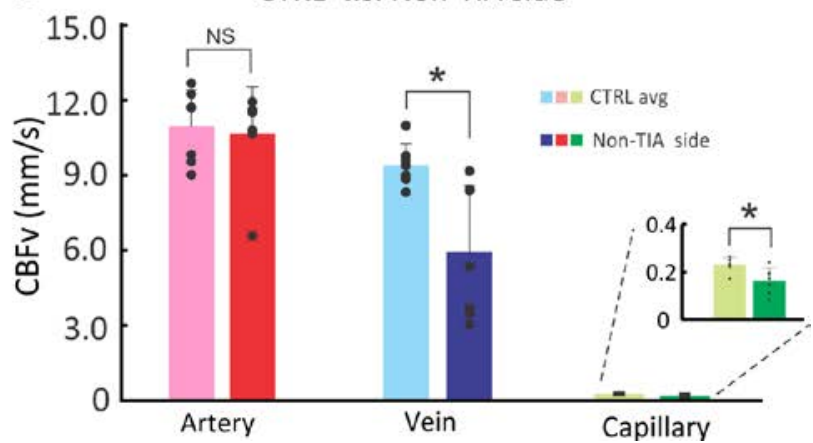

Vasoconstriction as a portent of TIA onset. Chronic cocaine is likely to elicit hypoperfusion in part by triggering vasoconstriction. In order to assess the contribution of vasoconstriction to TIA, we conducted vascular images using $\mu$ OCA. Figure 4 shows the corresponding $3 \mathrm{D}$ vasculature images $(\mu \mathrm{OCA})$ of Figure 3 , in which the vessel sizes of arterial and venous compartments of control and chronic cocaine mice are compared. Statistical analysis across different animals shown in Figure 4, B and C, indicate vasoconstriction in TIA mice. The analyses revealed that vasoconstriction occurred predominantly in the venous compartment. Specifically, the mean diameter of veins was most markedly decreased on the TIA-side from $72.45 \pm 11.06 \mu \mathrm{m}$ to $50.81 \pm 14.4 \mu \mathrm{m}(30.8 \% \pm 11.6 \%, P=0.008)$ and less so on the non-TIA side to 58.46 $\pm 11.26 \mu \mathrm{m}(19.7 \% \pm 5.7 \%, P=0.037)$. In contrast, vasoconstriction in arterial vessels was not significant, reduced by only $9.7 \% \pm 7.8 \%(P=0.385)$, from $38.66 \pm 9.34 \mu \mathrm{m}$ to $34.57 \pm 7.54 \mu \mathrm{m}$ on the TIA side and $4.3 \% \pm 15.6 \%(P=0.480)$ to $35.85 \pm 4.11 \mu \mathrm{m}$ on the non-TIA side.

It is noteworthy that, in contrast to the marked laterality in the CBFv decreases (Figure 3), the laterality differences in vasoconstriction, though showing some degree of diameter decrease on the TIA side compared with contralateral side $(5.7 \% \pm 11.2 \%$ for arteries, $17.94 \% \pm 13.19 \%$ for veins), were not significant.

The discrepancies between the association of flow decreases and vasocontraction for veins but not for arteries might reflect the fact that arterial stenosis can occur on a regionally localized section of the arterial vessel, whereas vasoconstriction of veins is more likely to be present throughout the entire length of the vessels. Indeed, the $\mu$ OCA reveals highly localized areas of stenosis in the arterial vessels (e.g., large yellow arrows in Figure 4A, left side of TIA panel), which appear as areas of flow discontinuity in the $\mu$ ODT images (yellow arrows in Figure 3A, left side of TIA panel). In addition, the local capillary networks near 
A

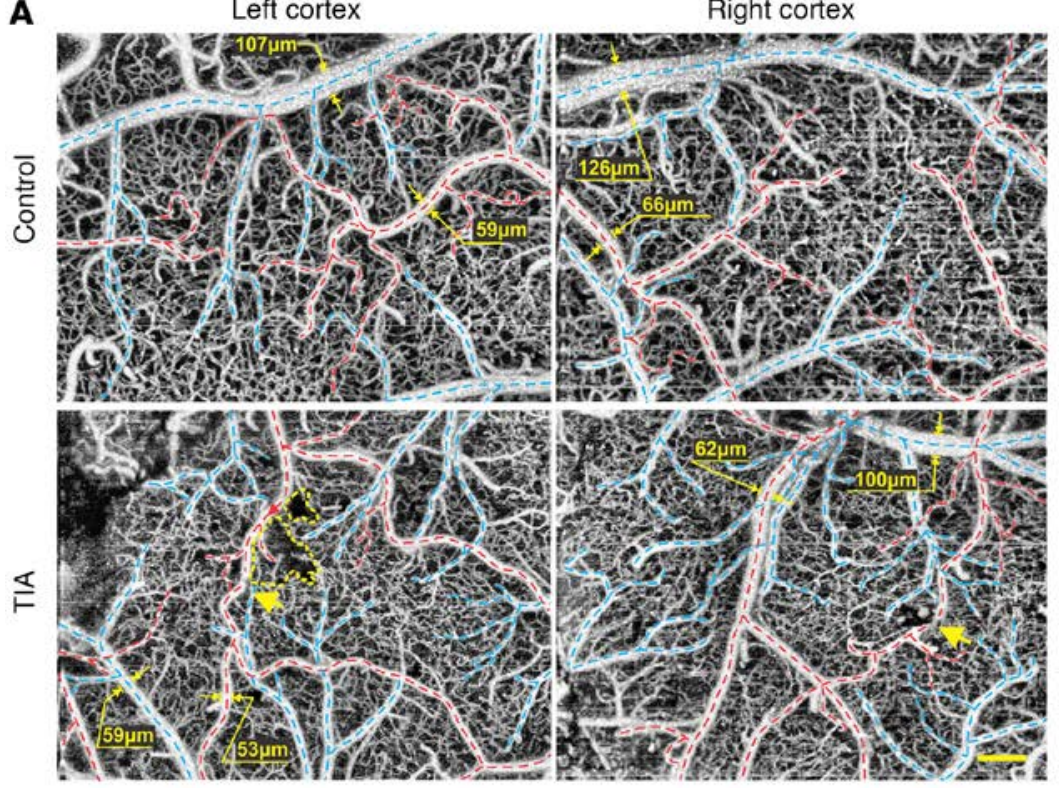

Figure 4. Cocaine elicited vasoconstriction of left and right sensorimotor cortices by $3 \mathrm{D} \mu \mathrm{OCA}$. (A) Cerebral vascular network of mouse sensorimotor cortices imaged by 3D $\mu$ OCA (upper row: control mouse; lower row: TIA mouse). Yellow arrows point to arterial stenosis, and dashed yellow lines outline the resultant disruption of local capillary networks. Dashed red/ blue lines: arterial/venous vessels. Scale bar: 200 $\mu \mathrm{m}$. (B and $\mathbf{C}$ ) Statistic figures of arterial and venous vessel sizes in left (TIA) and right (non-TIA) cortices, respectively. $n=7$ for each group. Unpaired 2-tailed Student's $t$ tests were performed for statistical analysis, and average values are shown as the mean \pm SD in B and C. ${ }^{*} P<0.05,{ }^{*} P<0.01$.
B

B

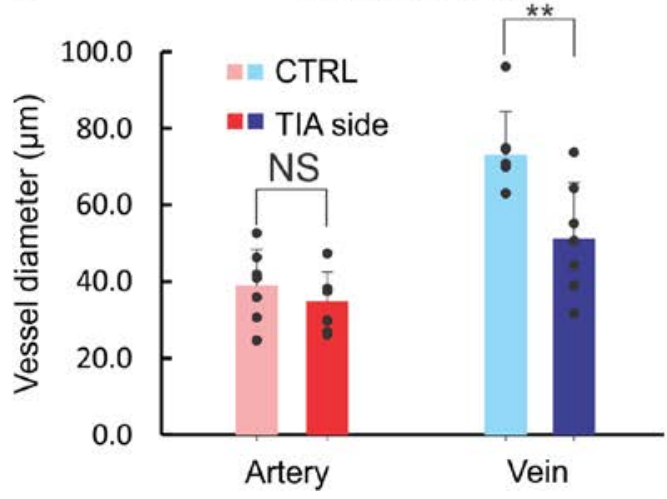

C

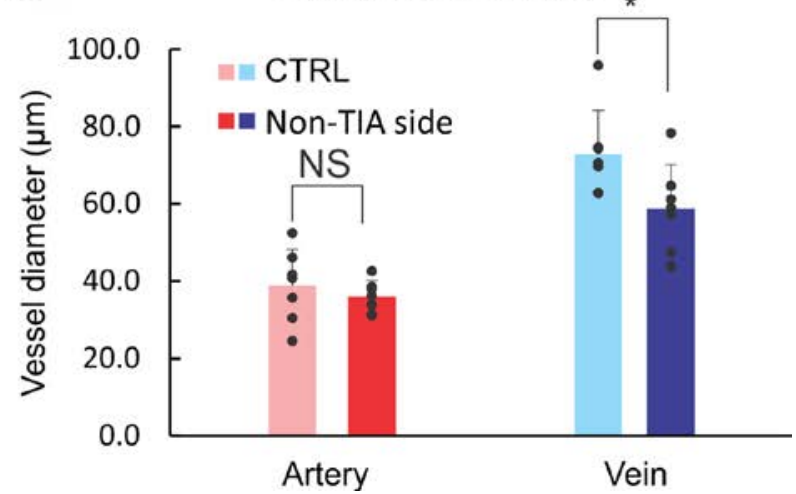

the arterial stenosis were disrupted, as shown in Figure 4A (e.g., the area marked by the yellow dished lines, left side of TIA panel). In contrast, the $\mu \mathrm{OCA}$ reveals narrowing of veins throughout the length of the vessel. The significant decreases in venous diameters in the chronically cocaine exposed mice is likely to reflect both adaptations to accommodate the reduced flow to the tissue to avoid inflow/outflow, as well as the direct vasoconstricting effects of cocaine to venous vessels $(13,14)$.

$C B F v$ dynamic response to acute cocaine challenge. Figure 1 reveals that the TIA onset, including the emergence of hemiparalysis in the chronic cocaine mice, was transient and occurred 2-3 minutes after acute cocaine $(35 \mathrm{mg} / \mathrm{kg}$, i.p.) recovering in $<20$ minutes. The occurrence of the TIA following an acute cocaine injection only after the mice had received repeated cocaine doses suggests that it is likely a compounding effect of both severe basal hypoperfusion (Figure 3) secondary to chronic vasoconstriction (Figure 4), plus the additional $\mathrm{CBF}$ disruption triggered by the acute cocaine injection. Thus, we assessed the normalized dynamic response of $\mathrm{CBFv}$ in chronically cocaine-exposed mice following an acute cocaine injection, i.e., $\Delta \operatorname{CBFv}(\mathrm{t})=\left[\mathrm{CBFv}(\mathrm{t})-\operatorname{CBFv}\left(\mathrm{t}_{\mathrm{b}}\right)\right] / \operatorname{CBFv}\left(\mathrm{t}_{\mathrm{b}}\right) \times 100 \%$, where $\operatorname{CBFv}\left(\mathrm{t}_{\mathrm{b}}\right)$ is the averaged $\mathrm{CBFv}$ value before the acute cocaine injection ( $\mathrm{t}_{\mathrm{b}} £ \mathrm{~min}$ ), and we compared them to the responses of control mice when given an acute cocaine injection. Figure 5 shows the dynamic CBFv responses of a control mouse (upper panel) vs. a TIA mouse (lower panel) to an acute cocaine injection ( $35 \mathrm{mg} / \mathrm{kg}$, i.p.), in which the time-lapse $\Delta C B F v$ in arteries (red traces), veins (blue traces), and capillaries (green traces) are plotted. As expected, $\triangle \mathrm{CBFv}$ decreased in both control and TIA mice in response to acute cocaine. However, quantitative analyses by linear least squares fits (dashed lines) show a $\sim 9.5$-fold steeper slope of the arterial $\triangle \mathrm{CBFv}$ decrease in the TIA mouse $(-11 \% / \mathrm{min})$ than in the control mouse $(-1.23 \% / \mathrm{min})$, indicating that the flow disruption in the 
A

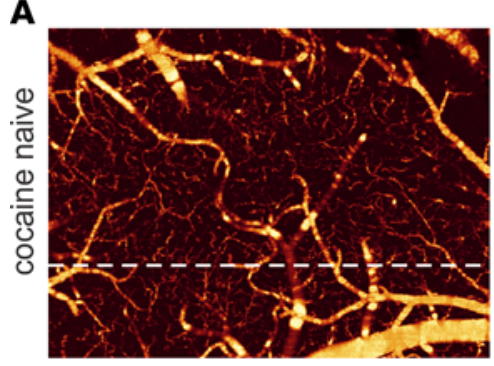

D

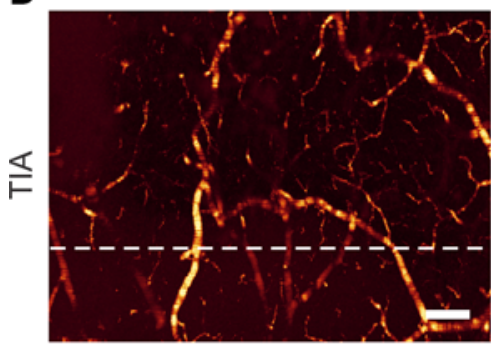

B

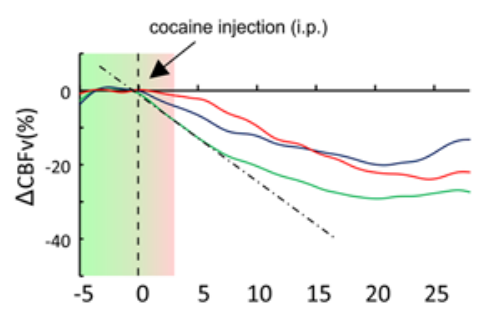

E

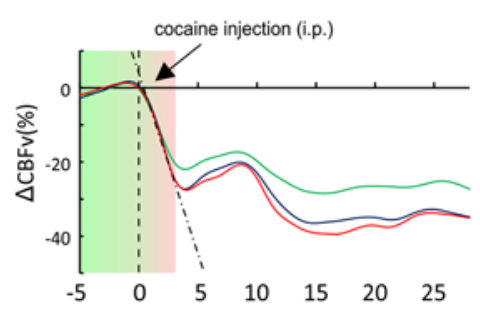

C
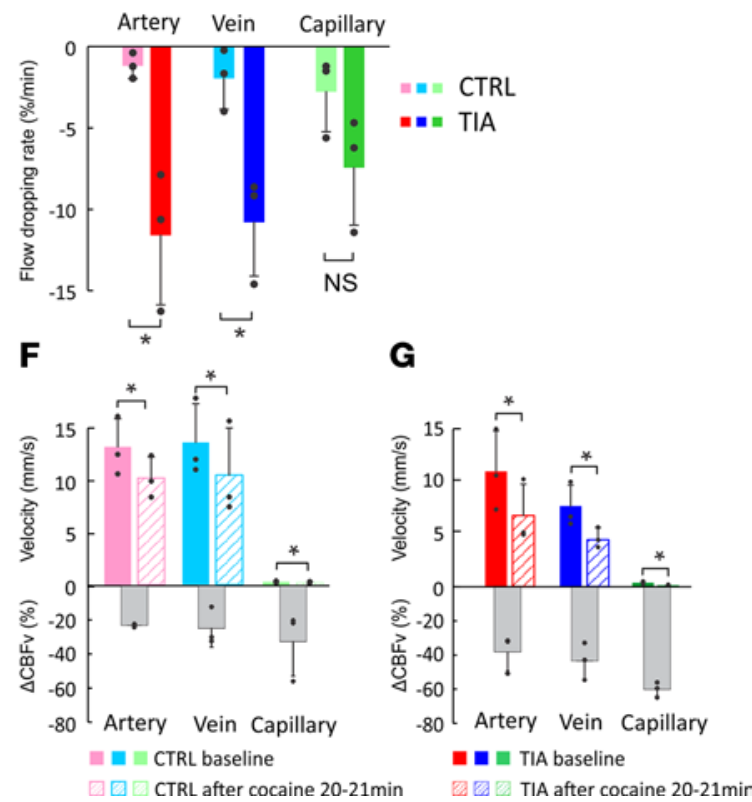

Figure 5. Comparison of CBFv dynamic responses to acute cocaine challenge between control and TIA mouse. (A and D) The baseline $\mu$ ODT image of control and cocaine mouse with B-scan position highlighted by white dashed line whose time-course curve are plotted in $\mathbf{B}$ and $\mathbf{E}$, containing multiple artery (red), vein (blue), and capillary (green). The black dashed lines in $\mathbf{B}$ and $\mathbf{E}$ are the linear curve fitting for artery flow decrease within the first 2.5 minutes after cocaine injection whose statistical comparison is presented in $\mathbf{C}$. The period shaded with green-to-red gradient represents the latency. Scale bar: $200 \mu \mathrm{m}$. (F and G) CBFv comparison of artery, vein, and capillary between pre- ( -5 to 0 min) and postinjection (20-21 min) session for control and cocaine-treated mice, respectively, in which the gray bars show relative decreases in flow (\%). Unpaired 2-tailed Student's $t$ tests were performed for $\mathbf{C}$, and paired $t$ tests were performed for $\mathbf{F}$ and $\mathbf{G}$. Average values are shown as the mean $\pm \mathrm{SD}$ in $\mathbf{C}, \mathbf{F}$, and $\mathbf{G}$. $n=3$ for each group. ${ }^{*} P<0.05$.

sensorimotor cortex of the TIA mouse was significantly faster than in the control mouse $(P=0.014$ and $P=$ 0.016 for arterial and venous flows, respectively). Interestingly, despite the basal hypoperfusion in TIA mice (Figure 2), they still show greater $\triangle \mathrm{CBF}$ decreases in response to acute cocaine than the control mice (in arteries, $-38.56 \%$ vs. $-22.97 \%$; veins, $-46.76 \%$ vs. $-24.82 \%$; and capillaries, $-60.55 \%$ vs. $-29.72 \%$ ). These results reveal that the low basal CBFv due to chronic cocaine is further disrupted by acute cocaine, which leads to an abrupt flow decrease that triggers the TIA and the associated hemiparalysis.

$C B F v$ reorganization in deep sensorimotor cortex. Figure 1 shows that cocaine-elicited TIA occurred most frequently within 17-24 days of chronic cocaine exposures, but then the incidence and severity decreased despite continuous cocaine daily exposures. Such intriguing behavioral patterns led us to investigate the underlying mechanisms that prevented TIA deterioration. For this purpose, we performed longitudinal in vivo $\mu \mathrm{ODT} / \mu \mathrm{OCA}$ imaging of mice every 4 days during chronic cocaine exposures through a chronic cranial window. Specifically, focal tracking was implemented to image both superficial layers (L1, L2/3: 0-300 $\mu \mathrm{m}$ ) and deep layers (L4, L5: 400-600 $\mu \mathrm{m}$ ) where thalamocortical inputs play a critical role in motor control $(15,16)$. Surprisingly, we observed that chronic cocaine elicited CBFv network reorganization across superficial and deep cortical layers, as shown in Figure 6. For control mice (left panels), $\mu \mathrm{ODT}$ images show almost no difference between day 8 and day 28 (e.g., high CBFv in superficial layers and low in deep layers). In contrast, in chronic cocaine mouse, the CBFv networks (e.g., in arterial, venous, and capillary vessels) in superficial layers were significantly decreased $(P=0.028, n=5)$, consistent with those shown in Figure 2 and Figure 3 that imaged superficial cortical layers, whereas the capillary $\triangle \mathrm{CBFv}$ (i.e., $\triangle \mathrm{CBFv}=\left[\mathrm{CBFv}-\mathrm{CBFv}_{\mathrm{b}}\right] / \mathrm{CBFv}_{\mathrm{b}} \times 100 \%$, where $\mathrm{CBFv}_{\mathrm{b}}$ is the flow rate on day 0 ) in deep layers was significantly increased $(P<0.001, n=5)$.

Statistical figures of time-lapse flow image analyses in Figure 6, C and D, indicate that the superficial $\mathrm{CBFv}$ networks in control mice remained largely unchanged. In chronic cocaine mice, superficial arterial $\triangle \mathrm{CBFv}$ (red trace) decreased to $-33.59 \% \pm 11.94 \%$ between day $17-32$, and then after cocaine withdrawal slowly recovered to $-21.41 \% \pm 3.85 \%$ at day 42 with no further changes thereafter. Although slightly 
A

Control

Superficial artery/vein CBFv (0-300)

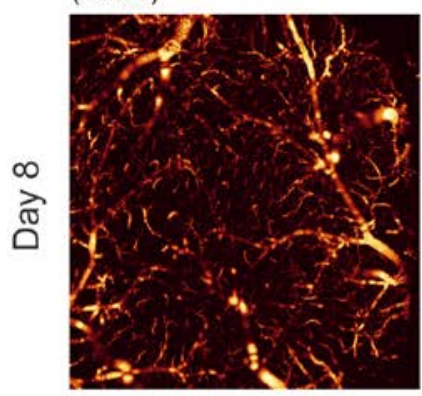

Deep brain $(300-600 \mu m)$
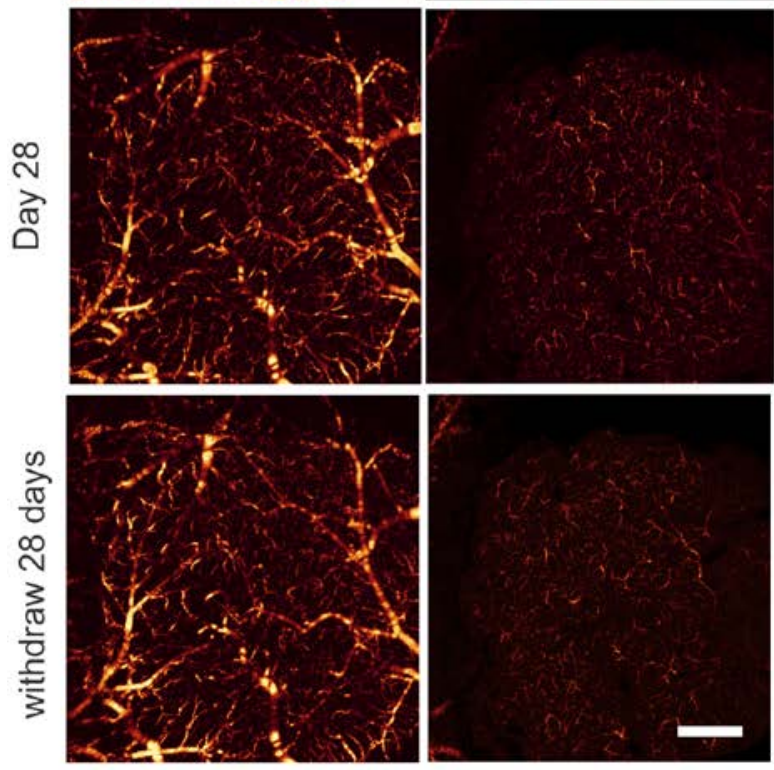

\section{C}

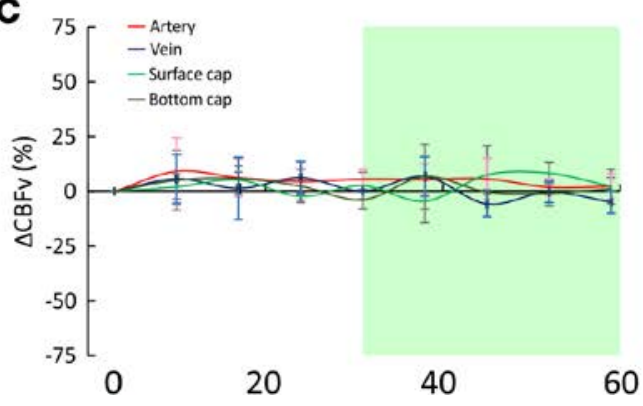

B

CC

Superficial artery/vein CBFv

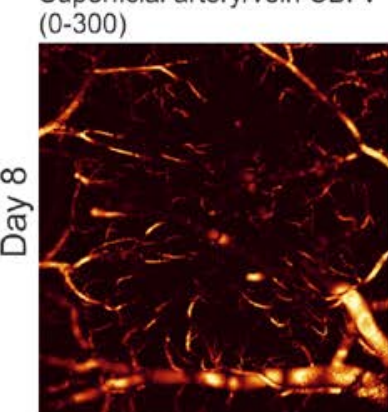

Deep brain $(300-600 \mu \mathrm{m})$
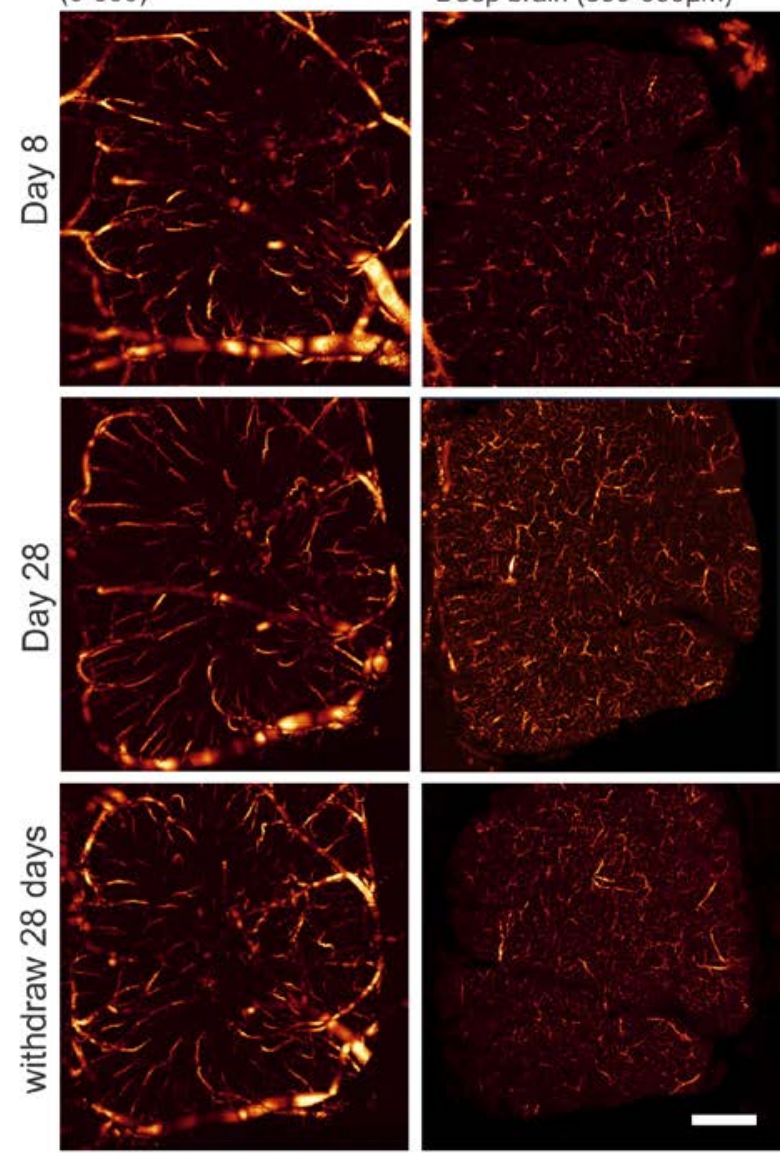

D

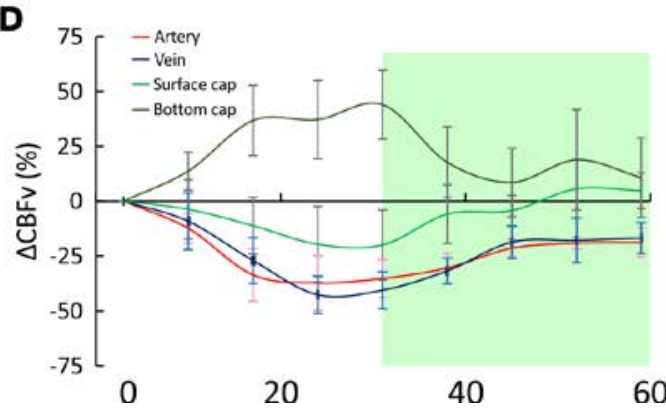

Figure 6. Chronic cocaine elicited CBFv reorganization in the sensorimotor cortex. (A) $\mu$ ODT images of superficial (A, C: L1, L2/3, 0-300 $\mu$ m) layers and deep cortical (B, D: L4, 300-600 $\mu \mathrm{m}$ ) layers of a control mouse acquired day 8 and day 28 through a chronic cranial window. (B) Mouse undergoing chronic cocaine exposures (35 mg/kg/day, i.p.). (C and D) Time courses of CBFv (\%) in arteries (red), veins (blue), superficial (light green), and deep (dark green) capillaries in control $(n=5)$ and chronic cocaine $(n=5)$ mice, respectively. CBFv change (C and $\mathbf{D})$ were tested for significant difference using one-way repeated measures ANOVA followed by a post-hoc test (Holm-Sidak method). Scale bar: $500 \mu \mathrm{m}$.

delayed, superficial venous $\triangle \mathrm{CBFv}$ (blue trace) also decreased to $-42.63 \% \pm 8.50 \%$ by day $22-32$ and then slowly recovered after cocaine withdrawal to $-18.66 \% \pm 7.36 \%$ at day 42 (partial recovery). Superficial capillary $\triangle \mathrm{CBFv}$ slowly decreased to $-19.64 \% \pm 17.29 \%$ around day $25-32$, and then after cocaine withdrawal, it almost recovered near day 38 followed by a slight overshoot $(P=0.935, n=5)$.

In contrast, deep capillary $\triangle \mathrm{CBFv}$ significantly increased $36.75 \% \pm 16.04 \%(P<0.001, n=5)$ by day 17 and remained high until day 32 , when cocaine was withdrawn. Afterward, it rapidly declined to $8.58 \%$ $\pm 15.62 \%(P=0.309, n=5)$ around day 42 but did not fully return to its baseline. Also, Figure 6B (day 28) 


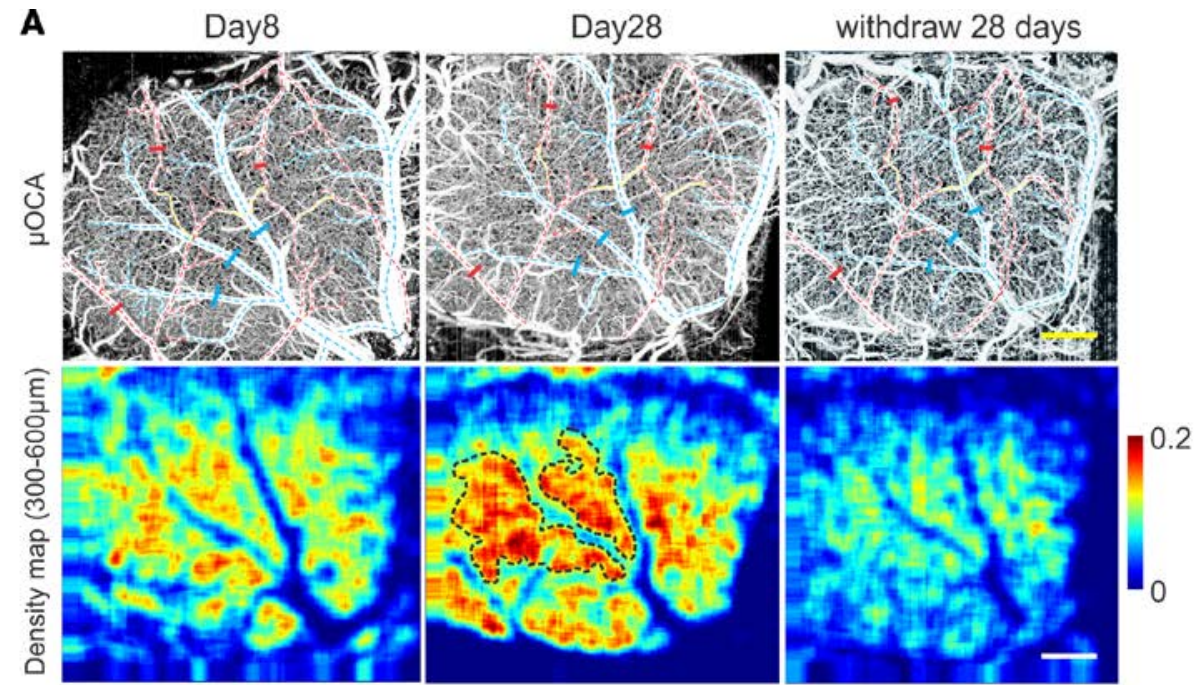

B

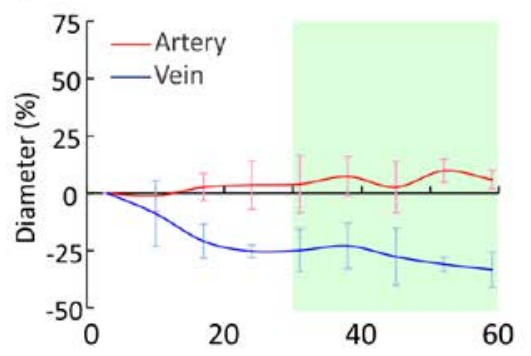

C

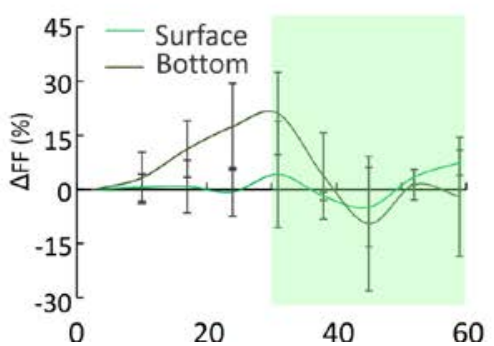

D

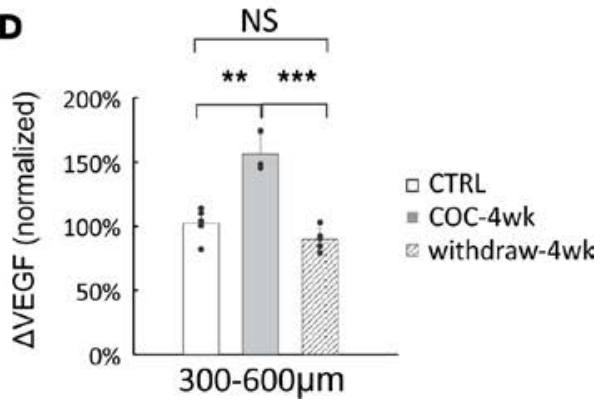

Figure 7. Cocaine elicited vasoconstriction in superficial layers and angiogenesis in deep layers of the sensorimotor cortex. (A) Upper row: cerebrovascular images acquired with $\mu$ OCA on day 0 and day 28 after cocaine exposures (35 mg/kg/day, i.p.); arterial and venous vessels are marked in red and blue dashed lines. Solid bars are marked for vessel size quantification. Lower row: corresponding microvasculature density maps of deep layers; dashed black circles outline the regions of most eminent angiogenesis surrounding areas with vessel vasoconstriction. (B) Time-lapse vasoconstriction in arteries and veins, (C) time-lapse microvascular density changes in superficial and deep cortical layers, and (D) VEGF upregulation. Vasoconstriction and angiogenesis (B and $\mathbf{C}$ ) were tested for significant difference using one-way repeated measures ANOVA followed by a post-hoc test (Holm-Sidak method) with $n=5$. Unpaired 2-tailed Student's $t$ tests were performed for statistical analysis, and average values are shown as the mean \pm SD in $\mathbf{D}$, with $n_{\text {ctrl }}=5, n_{4 \mathrm{w}-\mathrm{cc}}=3$, and $n_{\text {withdraw }}=5 .{ }^{* *} P<0.01,{ }^{* *} P<0.001$. Scale bar: $500 \mu \mathrm{m}$.

reveals that many vascular flow sections are longer (pointed out by arrows in Supplemental Figure 1), which is consistent with an increase of flow routing to the deep cortical layers.

Angiogenesis and VEGF upregulation. We hypothesized that hypoxia-driven angiogenesis, which is a well-documented process in stroke and in the tumor microenvironment, underlie the slow recovery and reorganization of flow observed with protracted cocaine exposure. Consistent with this hypothesis, we show in Figure 7 that chronic cocaine-induced vasoconstriction of arteries and veins ( $\mu \mathrm{OCA}$ day 28 vs. day 8) occurred in parallel to an increase in microvascular density, i.e., angiogenesis in deep cortical layers (density map day 28 vs. day 8). Quantitative analysis (see Supplemental Figures 2-4 for details) based on image segmentation and vessel tracking (17) showed (Figure 7C) that between day 0 and day 31 , there were no significant changes in microvascular density in superficial layers $(=4.18 \% \pm 14.72 \%, P$ $=0.484$; green trace) but there were significant increases $(21.04 \% \pm 11.98 \%, P=0.006)$ in deep cortical layers (dark trace). The increases in vascular density in the deep cortical layers correlated with the time of greatest vasoconstriction in the venous vessels (blue trace in Figure 7B), suggesting that angiogenesis following chronic cocaine exposure was triggered by vasoconstriction-induced ischemia. This was further cross-validated by ex vivo VEGF fluorescence microscopy, which showed significant VEGF upregulation in the deep cortical layers $(152.50 \% \pm 15.56 \%, P=0.002)$ after 4 -week cocaine exposures and a fallback to $87.73 \% \pm 8.71 \%(P=0.105)$ after 28 days of cocaine withdrawal. Interestingly VEGF was also significantly increased in superficial layers $(202.83 \% \pm 83.64 \%, P=0.032)$ at 4 weeks and then fell back to $99.89 \% \pm$ $27.90 \%(P=0.995)$ after 28 days of withdrawal. 
A. $0.9 \%$ Saline Injection $(0.7 \mathrm{cc} / 100 \mathrm{~g} /$ day i.p. $)+$ behavior recording
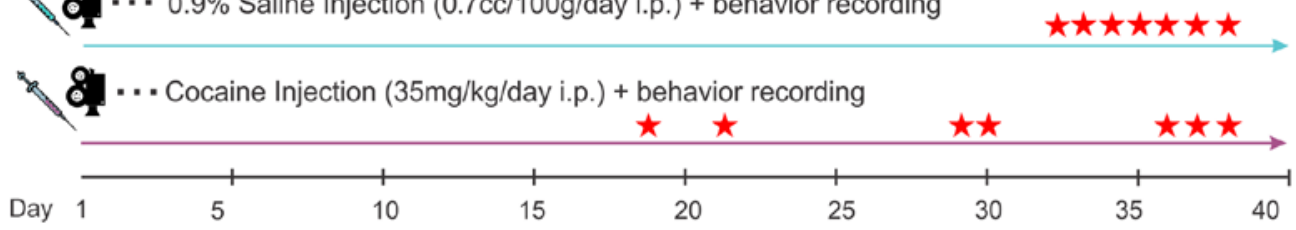

B

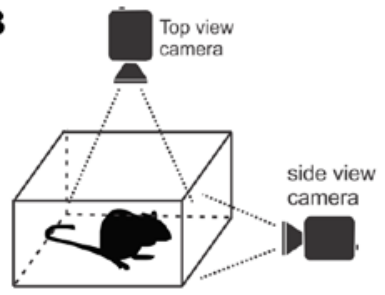

Video Recording( $\sim 40$ mins/mouse/day)

$\sim 5 \mathrm{~min}$ baseline $+\sim 35 \mathrm{~min}$ after cocaine

represents the surgery and image day for one mouse in each group.

C

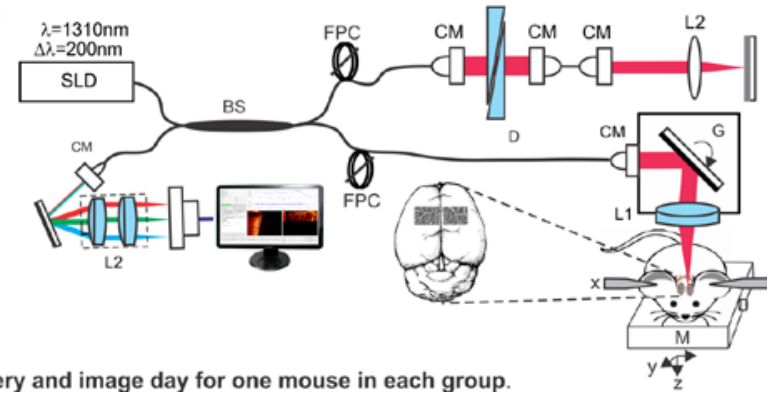

Figure 8. Experimental design used to study cocaine-induced transient ischemic attack (TIA). (A)Fourteen C57BL/6) mice were split into 2 groups, control $(n=7)$ and chronic cocaine $(n=7)$ group, which were treated daily with $0.9 \%$ saline and with $35 \mathrm{mg} / \mathrm{kg}$ cocaine (i.p.), respectively. (B) The behavioral motor responses were measured 3 min before injection (used as baseline) and $30 \mathrm{~min}$ after injection using a dual-perspective camera system. (C) In vivo imaging by $\mu \mathrm{ODT} / \mu \mathrm{OCA}$ was conducted within 24 hours after the first appearance of hemiparalysis (described as TIA phase II).

\section{Discussion}

In this paper, we show that in chronically cocaine-exposed mice, the abrupt exacerbation of vasoconstriction and hypoperfusion triggered by an acute cocaine challenge led to cortical ischemia and the emergence of a TIA. We also show that chronic cocaine exposure gradually resulted in a redistribution of flow from superficial to deep cortical layers and led to angiogenesis in deep cortical layers that, we hypothesize, prevented further deterioration of the TIA with continuous cocaine use. This study, for the first time to our knowledge, shows evidence of a TIA in an animal model of chronic cocaine exposure that allowed us to investigate the longitudinal changes in the cerebrovascular networks associated with TIA with protracted cocaine exposure. This allowed us to show that: (i) chronic cocaine exposure induces severe vasoconstriction and hypoperfusion, which is exacerbated during acute intoxication, triggering TIAs that presented as transitory hemiparalysis, and (ii) vascular adaptations including CBFv reorganization from superficial to deep cortical layers along with angiogenesis prevent further exacerbation of TIA with continued cocaine exposures.

Our results corroborate prior findings from our group that chronic cocaine enhances the vasoconstricting effects and the decreases in CBFv triggered by an acute cocaine challenge, thus leading to ischemia (18). Here, we expand our understanding by documenting that this sensitized vascular response is responsible for the emergence of the TIA.

Furthermore, our longitudinal design allowed us to monitor for the first time to our knowledge the emergence and severity of the TIA as a function of days of cocaine exposure, and showed that while TIA severity peaked around 17-24 days, it declined thereafter, which was suggestive of a compensatory response to counteract the hypoperfusion.

The quantitative capability of our imaging tools with their high temporal and spatial resolutions allowed us to monitor dynamic changes in the cerebrovascular networks. We found the CBFv was decreased in chronic cocaine-induced animals across the vascular network, including arteries, veins, and capillaries, which is in agreement with clinic finding of CBF reduction in cocaine abusers (19). In addition, we observed evidence of arterial stenosis in the cortex of TIA animals that was associated with reduced $\mathrm{CBFv}$ in microvascular networks in the surrounding local area (Figure 3 and Figure 4). Though we are interpreting the reductions in $\mathrm{CBFv}$ to reflect arterial vasoconstriction, we cannot rule out the contribution of microthrombosis. Indeed, clinical studies have reported both vascular stenosis (20) and microthrombosis in cerebral and coronary arteries of cocaine abusers $(21,22)$. The parallel time-lapse imaging allowed us to assess the associations between TIA hemiparalysis and CBFv disruption elicited 
by chronic cocaine exposure. Though prior studies had shown decreases in cortical CBF (23) in cocaine abusers and in animal models of chronic cocaine exposures, the enhanced spatial resolution of our imaging tools allowed us to quantify flow in various vascular compartments and to link these, for the first time to our knowledge, with the associated behavioral deficits. Noteworthy was the marked reduction of flow in capillaries ( $67 \%$ reduction on the TIA side and $30 \%$ on the non-TIA side) prior to the acute cocaine challenge. This marked flow deficit in the resting state could be regarded as a portent for TIA, particularly when further challenged with an acute dose of cocaine.

Our findings indicate that the TIA is triggered by both pronounced and sustained CBFv decreases from the chonic cocaine exposure, along with an abrupt exacerbation of the CBFv decreases during intoxication ( $\sim 9.5 \times$ steeper flow drop in response to an acute cocaine challenge). Although decreases in resting-state flow could result in impaired neuronal function due to the tight coupling between neuronal activity and $\mathrm{CBF}$, these resting flow reductions by themselves did not account for the TIA paralysis, which manifested only after an acute cocaine exposure. We postulate that this abrupt decrease in CBFv interferes with the buffering capacity of the vascular system to compensate for regional hypoperfusion. Indeed, abrupt perturbation of CBF can trigger symptom presentation in vascular diseases in other organs, such as lung and heart $(24,25)$.

Intriguingly, motor behavior in the chronic cocaine-exposed mice recovered within $14^{\prime} 28^{\prime \prime} \pm 20^{\prime} 3$ after an acute cocaine challenge, even though they still had marked deficits in arterial $(38.56 \% \pm 11.00 \%)$, venous $(43.88 \% \pm 10.89 \%)$ and capillary $(60.55 \% \pm 4.44 \%)$ perfusion. This is suggestive of an uncoupling between neurophysiological and cerebral vascular deficits, which is a phenomenon that has previously been reported clinically in disorders associated with cerebral ischemia $(26,27)$.

Our findings revealing flow redistribution from superficial to deep cortical layer, increases in vessel density, and increases in VEGF suggest that these compensatory responses might have prevented further deterioration of the TIA despite continuous cocaine use. Ischemia triggered by cocaine is likely to have stimulated angiogenesis, as has been shown to be the case for animal models of cerebral stroke (28). Indeed, prior studies showed that chronic cocaine elicited marked oxyhemoglobin $\left(\mathrm{HbO}_{2}\right)$ decreases, along with an upregulation of VEGF and HIF that were interpreted to reflect hypoxia-driven angiogenesis (29). Similarly, upregulation of platelet-derived growth factor (PDGF) after chronic cocaine was interpreted to reflect an increase in vessel permeability that might indirectly stimulate angiogenesis (30). Apart from ischemia, it is also plausible that the serotonin-enhancing effects of cocaine might have also contributed to angiogenesis for sustained administration of selective serotonin reuptake inhibitors (SSRI), which have been shown to improve recovery of stroke in animal models and in humans $(31,32)$. Inasmuch as cocaine, like SSRI, blocks serotonin transporters $(33,34)$, this effect could have accelerated the recovery associated with its vasoconstricting effects.

Additionally, collateral circulation determines the fate of salvageable ischemic tissue, and its augmentation is widely regarded as a promising therapeutic approach to stroke or ischemia. However, technical limitations of image resolution or depth of prior imaging studies of TIA had prevented the assessment of flow across different cortical layers. Here, we were able to longitudinally image CBFv networks across cortical L1-L6 in chronic cocaine mice for 9 weeks (5-week cocaine treatment and 4-week withdrawal). The observed $36.75 \% \pm 16.04 \%$ increase of capillary CBFv in deep cortical layers (L3-L5: 300-600 $\mu \mathrm{m}$ ) was likely due to enhanced collateral circulation to compensate for flow deficits. The reorganization of $\mathrm{CBF}$ from superficial to deep cortical layers in the somatosensory cortex (35) was associated with longer vessels reaching the deep cortical layers and with significant increases in VEGF. Vascular networks across cortical layers are heterogeneous (36); thus, this could account for the differences in the vascular responses to chronic cocaine between the superficial and the deep cortical layers. However, the flow redistribution could also reflect differences in neuronal demand needs, vascular innervation, or collateral perfusion between the deep and the superficial cortical layers (37). Alternatively, they could reflect differences in neuronal sensitivity to cocaine, as shown by the higher sensitivity of superficial layers to cocaine's enhancement of sensory signals than that of deep cortical layers (38).

The vascular adaptations that we report in our model of chronic cocaine exposure are analogous to those reported in animal models of arterial occlusion (39), which benefited from proangiogenic therapies. This would suggest that similar strategies might be beneficial to help enhance brain recovery after cocaine-induced ischemia $(40,41)$. Thus, our findings are clinically relevant, which suggests that enhancing angiogenesis might prevent strokes in cocaine abusers and improve recovery in cocaine abusers suffering 
from strokes or TIA presentations. Similarly, it suggests that other pharmacological interventions shown to be beneficial in enhancing recovery from stroke, such as SSRI, might also help prevent and/or accelerate recovery from ischemia in cocaine abusers. Additionally, the characterization of the development of TIA and emerging angiogenesis with repeated cocaine exposure provides a model with which to test therapeutic strategies to minimize cocaine-induced hypoxia that could be clinically beneficial.

In interpreting our findings, it is important to address its limitations, most notable of which is the limitation of translating findings in an animal model to the human condition, which is a universal confound in preclinical studies. Indeed, cocaine abusers frequently consume multiple drugs (i.e., nicotine, alcohol) and might inject contaminants (i.e., procainamide), all of which can contribute to cocaine-associated strokes and TIA, whereas we only administered cocaine to our animals. However, this - rather than detract - enhances the value of our findings because it indicates that cocaine by itself is able to induce cerebrovascular pathology, which in humans might be further exacerbated by comorbid use of other drugs or contaminants. Also, while multiple studies have given cocaine to rodents, this is the first one to report a consistent emergence of a TIA with chronic cocaine, which raises the possibility that the strain of mice (C57/B6 mice) was particularly sensitive to cocaine-induced vascular dysfunction and calls into question its generalizability to other strains. However, this is not different from the diversity seen clinically. Indeed, cerebrovascular pathology does not occur in all cocaine abusers, even in those with very severe levels of abuse, which is likely to reflect intersubject diversity (genetic, developmental, environmental) that leads to differences in cerebrovascular responses to cocaine. Moreover, one could argue that an animal model that has greater sensitivity to cocaine's adverse cerebrovascular effects offers a tool with which to investigate the development and recovery of cocaine-induced TIA. Nonetheless, as for other conditions for which animal models are used to study diseases, these have to be recognized as tools that help us control and measure processes that are not possible to do in humans but that don't always have a direct one-to-one correspondence to human diseases. For our study, we chose a daily cocaine dose of $35 \mathrm{mgkg}$ (i.p.) since this dose results in cocaine plasma levels consistent with those that have been reported in cocaine abusers (42). However, since in our study we did not measure cocaine levels in plasma nor its metabolites, this limits our ability to determine the representativeness of the cocaine levels in our mice compared with those seen clinically. Also, the failure to measure cocaine and its metabolites in plasma made us assess if these changed with chronic exposure and whether they related to the observed recovery. However, note that - at least for 7 days - exposure rodent studies showed that cocaine plasma concentrations did not change between acute and chronic cocaine exposures ( 7 days, $20 \mathrm{mg} / \mathrm{kg}$, i.p.) (42). Finally, a limitation for our studies was that the imaging was conducted under anesthesia (isoflurane), which might have influenced the reactivity of blood vessels to cocaine. We selected isoflurane anesthesia to minimize these potential confounding effects, since it does not alter PCO2 (e.g., arterial carbon dioxide tension) nor PO2 (e.g., coronary sinus oxygen tension) (43).

In summary, here we show that chronic cocaine decreased CBF and sensitized vessels to cocaine-induced vasoconstriction such that, with an acute cocaine injection, this triggered an abrupt reduction in flow and ischemia, resulting in a TIA manifested as contralateral paralyses. We also show that vasoconstriction and ischemia triggered by cocaine were associated with vascular reorganization and angiogenesis that we hypothesize prevented further deterioration of TIA, despite continuous cocaine administration. Strategies to accelerate vascular recovery might be beneficial to improve clinical outcomes in cocaine abusers.

\section{Methods}

Animals and pharmacological treatments. A total of 24 C57/B6 mice (male, 12-14 weeks of age, The Jackson Laboratory) were divided into two groups, controls and chronic cocaine groups, in which animals received daily i.p. injection of $0.9 \%$ saline $\left(0.7 \mathrm{cc} / 100 \mathrm{~g} / \mathrm{day}, n_{\mathrm{ct}}=12\right)$ or cocaine $\left(35 \mathrm{mg} / \mathrm{kg} / \mathrm{day}, n_{\mathrm{cc}}=12\right)$ for 34 consecutive days. We used this dose because it resulted in cocaine plasma levels consistent with those observed in cocaine abusers (42). For the acute cocaine challenge procedure, animals received either an injection of $0.9 \%$ saline $(0.7 \mathrm{cc} / 100 \mathrm{~g}$, i.p.) or cocaine $(35 \mathrm{mg} / \mathrm{kg}$, i.p.).

Surgery. Mice were anesthetized with a mixture of $2.0 \%-2.5 \%$ isoflurane (Henry Schein Animal Health) in $100 \%$ oxygen. Then, their heads were firmly affixed on a custom stereotaxic frame to minimize motion artifacts. In 7 control and 7 chronic cocaine mice, we placed 2 cranial windows $(\sim 2.5 \times$ $\left.2.5 \mathrm{~mm}^{2}\right)$ above the left ( 0.25 to 2.75 lateral and -0.25 to -2.75 anterior to bregma) and the right $(-0.25$ to -2.75 lateral and -0.25 to -2.75 anterior to bregma) sensorimotor cortices, and in 5 control and 5 
chronic cocaine mice, we placed 1 cranial window above the left sensorimotor cortex. The exposed cranial surface regions were covered with $2 \%$ agarose gel and affixed with $100 \mathrm{~m}$-thick glass coverslip using biocompatible cyanocrylic glue. The physiological state of the mice, including ECG, respiration rate, and body temperature, was continuously monitored (SA Instruments).

Behavioral recording. A behavioral recording chamber consisting of two 720 pixel color video cameras (Canon VIXIA HF S21) placed orthogonally from top and one side of an uncovered rat cage was employed to record locomotor behavior. Locomotor behavior was recorded daily starting 5 minutes prior to saline $(0.7 \mathrm{cc} / 100 \mathrm{~g}$, i.p.) or cocaine $(35 \mathrm{mg} / \mathrm{kg}$, i.p.) injection (baseline period) and followed for 35 minutes after injection (postinjection session). A total of 7 control and 7 chronic cocaine mice were recorded (see Table 1$)$.

$\mu O D T / \mu O C A$ imaging. A custom $\mu \mathrm{OCT}$ system was used to acquire $3 \mathrm{D}$ cross-sectional dynamic images of the sensorimotor cortex through the cranial window over a relatively large field of view (e.g., FOV $>2 \times 2 \times 1 \mathrm{~mm}^{3}$ ) and at up to $147 \mathrm{kHz}$ axial scan rate (Supplemental Figure 5). The axial resolution of the $\mu \mathrm{OCT}$ image - determined by the coherence length $\left(L_{c}=2 \ln 2 / \pi \times \lambda^{2} / \Delta \lambda_{c p}, \lambda=1,310 \mathrm{~nm}\right.$, cross-spectrum bandwidth $\Delta \lambda_{\mathrm{cp}} \approx 200 \mathrm{~nm}$ ) of an ultra-broadband superluminescence diode (Thorlabs) - is 2.8 $\mu \mathrm{m}$, and its transverse resolution is $3.2 \mu \mathrm{m}$ as determined by the focal spot size of the microscope objective (f18mm/NA0.25, Edmunds optics). Graphic processing unit-powered software (NVIDIA, Geforce GTX 670) for accelerating fast Fourier transform (FFT) image reconstruction and processing (6) was implemented to facilitate immediate display of microvascular morphology (3D $\mu \mathrm{OCA})$ and blood flow velocity networks (3D $\mu \mathrm{ODT}$ ) at a B-scan updating rate of up to 473 fields per second (fps) (2 M pixels/s), a feature that is useful for locating regions of interest (ROIs) with disrupted/constricted vessels or microsichemia during image acquisition. Figure 8 illustrates the protocols for recording both behavioral videos and $\mu \mathrm{ODT} / \mu \mathrm{OCA}$ image sets during chronic cocaine administration.

Statistics. Statistical tests were performed with SYSTAT Software. Sample size for all in vivo experiments was determined using experimental data from other studies to approximate the number of mice necessary to give $>85 \%$ confidence for a two-fold change in any given parameter at the $P<0.05$ significance level. Differences of CBFv, vessel diameter, flow decrease slope, and VEGF upregulation were tested by 2-tailed $t$ tests or rank sum test. Rest-state flow differences before and after cocaine challenge of control was tested by paired t-test, and the differences of CBFv decline between control and TIA mice was tested by unpaired t-test. Correlation coefficients between cortical hypoperfusion and locomotion were calculated using Pearson statistics. In the chronic window study, $\mathrm{CBFv}$, vasoconstriction, and microvascular density were tested for significant difference using one-way repeated measure ANOVA followed by a post-hoc test (Holm-Sidak method). ${ }^{*} P<0.05 ;{ }^{* *} P<0.01,{ }^{* * *} P<0.001$. All data are presented as mean $\pm \mathrm{SD}$

Study approval. Animal-related procedures used in this study were carried out according to the Animal Use Protocol 230767, which was reviewed and approved by the Institutional Animal Care and Use Committee of Stony Brook University.

\section{Author Contributions}

YP, CD, and NDV designed the study. JY carried out and analyzed in vivo images. QZ conducted cocaine treatment and behavior recording. KP performed animal surgeries. KC carried out VEGF analyses. JY, NDV, CD, and YP wrote the paper. All authors proofread the manuscript.

\section{Acknowledgments}

This work was supported in part by NIH grants 1R01DA029718 (CD and YP), R21DA042597 (YP and CD), and NIH's Intramural Program of NIAAA (NDV).

Address correspondence to: Yingtian Pan, G17 Bioengineering Building, Stony Brook University, Stony Brook, New York 11794-5281, USA. Phone: 631.632.1519; E-mail: yingtian.pan@stonybrook.edu. Or to: Congwu Du, Department of Biomedical Engineering, Stony Brook University, 002 Life Science Building, Stony Brook University, Stony Brook, New York 11794-5281, USA. Phone: 631.632.5480; Email: congwu.du@stonybrook.edu. Or to: Nora D. Volkow, National Institute on Drug Abuse, Room 5274, 6001 Executive Boulevard, Rockville, Maryland 20852, USA. Phone: 301.443.6480; E-mail: nvolkow@nida.nih.gov. 
1. Lange RA, Hillis LD. Cardiovascular complications of cocaine use. N Engl J Med. 2001;345(5):351-358.

2. Satran A, et al. Increased prevalence of coronary artery aneurysms among cocaine users. Circulation. 2005;111(19):2424-2429.

3. Treadwell SD, Robinson TG. Cocaine use and stroke. Postgrad Med J. 2007;83(980):389-394.

4. Toossi S, Hess CP, Hills NK, Josephson SA. Neurovascular complications of cocaine use at a tertiary stroke center. J Stroke Cerebrovasc Dis. 2010;19(4):273-278.

5. Archavlis E, Carvi Y Nievas M. Cerebral vasospasm: a review of current developments in drug therapy and research. JPharm Technol Drug Res. 2013; 2(7):18.

6. Ren H, Du C, Yuan Z, Park K, Volkow ND, Pan Y. Cocaine-induced cortical microischemia in the rodent brain: clinical implications. Mol Psychiatry. 2012;17(10):1017-1025.

7. Pereira J, et al. Platelet activation in chronic cocaine users: effect of short term abstinence. Platelets. 2011;22(8):596-601.

8. Sáez CG, et al. Increased number of circulating endothelial cells and plasma markers of endothelial damage in chronic cocaine users. Thromb Res. 2011;128(4):e18-e23.

9. Daras M, Tuchman AJ, Marks S. Central nervous system infarction related to cocaine abuse. Stroke. 1991;22(10):1320-1325.

10. Sakadžić S, Lee J, Boas DA, Ayata C. High-resolution in vivo optical imaging of stroke injury and repair. Brain Res. 2015;1623:174-192.

11. Jia Y, Grafe MR, Gruber A, Alkayed NJ, Wang RK. In vivo optical imaging of revascularization after brain trauma in mice. Microvasc Res. 2011;81(1):73-80.

12. Srinivasan VJ, et al. Multiparametric, longitudinal optical coherence tomography imaging reveals acute injury and chronic recovery in experimental ischemic stroke. PLoS One. 2013;8(8):e71478.

13. Sofuoglu M, Nelson D, Babb DA, Hatsukami DK. Intravenous cocaine increases plasma epinephrine and norepinephrine in humans. Pharmacol Biochem Behav. 2001;68(3):455-459.

14. Abbound FM, Eckstein JW, Zimmerman BG, Graham MH. Sensitization of arteries, veins, and small vessels to norepinephrine after cocaine. Circ Res. 1964;15:247-257.

15. Yu X, et al. Direct imaging of macrovascular and microvascular contributions to BOLD fMRI in layers IV-V of the rat whisker-barrel cortex. Neuroimage. 2012;59(2):1451-1460.

16. Yu X, Qian C, Chen DY, Dodd SJ, Koretsky AP. Deciphering laminar-specific neural inputs with line-scanning fMRI. Nat Methods. 2014;11(1):55-58.

17. Frangi AF, Niessen WJ, Vincken KL, Viergever MA. In: Wells WM, Colchester A, Delp S eds. Medical Image Computing and Computer-Assisted Interventation — MICCAI'98: First International Conference Cambridge, MA, USA, October 11-13, 1998 Proceedings (Lecture Notes in Computer Science). Berlin, Heidelberg: Springer Berlin Heidelberg; 1998:130-137.

18. Ren H, Du C, Yuan Z, Park K, Volkow ND, Pan Y. Cocaine-induced cortical microischemia in the rodent brain: clinical implications. Mol Psychiatry. 2012;17(10):1017-1025.

19. Volkow ND, Mullani N, Gould KL, Adler S, Krajewski K. Cerebral blood flow in chronic cocaine users: a study with positron emission tomography. Br J Psychiatry. 1988;152:641-648.

20. Ibrahim M, Hasan R, Awan M. Cocaine-induced coronary stent thrombosis. Exp Clin Cardiol. 2013;18(1):e57-e59

21. Konzen JP, Levine SR, Garcia JH. Vasospasm and thrombus formation as possible mechanisms of stroke related to alkaloidal cocaine. Stroke. 1995;26(6):1114-1118.

22. Killam AL. Cardiovascular and thrombosis pathology associated with cocaine use. Hematol Oncol Clin North Am. 1993;7(6):1143-1151.

23. Volkow ND, Rosen B, Farde L. Imaging the living human brain: magnetic resonance imaging and positron emission tomography. Proc Natl Acad Sci USA. 1997;94(7):2787-2788.

24. Perper JA, Van Thiel DH. In: Galanter M ed. Recent Developments in Alcoholism: Alcohol and Cocaine Similarities and Differences Clinical Pathology Psychosocial Factors and Treatment Pharmacology and Biochemistry Medical Complications. Boston, MA: Springer US; 1992:363-377.

25. Schwartz BG, Rezkalla S, Kloner RA. Cardiovascular effects of cocaine. Circulation. 2010;122(24):2558-2569.

26. Rossini PM, et al. Does cerebrovascular disease affect the coupling between neuronal activity and local haemodynamics? Brain. 2004;127(Pt 1):99-110.

27. Liu YH, et al. Assessment of neurovascular dynamics during transient ischemic attack by the novel integration of micro-electrocorticography electrode array with functional photoacoustic microscopy. Neurobiol Dis. 2015;82:455-465

28. Font MA, Arboix A, Krupinski J. Angiogenesis, neurogenesis and neuroplasticity in ischemic stroke. Curr Cardiol Rev. 2010;6(3):238-244.

29. Krock BL, Skuli N, Simon MC. Hypoxia-induced angiogenesis: good and evil. Genes Cancer. 2011;2(12):1117-1133.

30. Yao H, Duan M, Buch S. Cocaine-mediated induction of platelet-derived growth factor: implication for increased vascular permeability. Blood. 2011;117(8):2538-2547.

31. Siepmann T, et al. Selective serotonin reuptake inhibitors to improve outcome in acute ischemic stroke: possible mechanisms and clinical evidence. Brain Behav. 2015;5(10):e00373.

32. Siepmann T, et al. The Effects of Pretreatment versus De Novo Treatment with Selective Serotonin Reuptake Inhibitors on Short-term Outcome after Acute Ischemic Stroke. J Stroke Cerebrovasc Dis. 2015;24(8):1886-1892.

33. Ravna AW, Sylte I, Dahl SG. Molecular mechanism of citalopram and cocaine interactions with neurotransmitter transporters. J Pharmacol Exp Ther. 2003;307(1):34-41.

34. Thompson BJ, et al. Transgenic elimination of high-affinity antidepressant and cocaine sensitivity in the presynaptic serotonin transporter. Proc Natl Acad Sci USA. 2011;108(9):3785-3790.

35. You J, Zhang Q, Park K, Du C, Pan Y. Quantitative imaging of microvascular blood flow networks in deep cortical layers by $1310 \mathrm{~nm} \mu$ ODT. Opt Lett. 2015;40(18):4293-4296.

36. Merkle CW, Srinivasan VJ. Laminar microvascular transit time distribution in the mouse somatosensory cortex revealed by Dynamic Contrast Optical Coherence Tomography. Neuroimage. 2016;125:350-362.

37. Jackman K, Iadecola C. Neurovascular regulation in the ischemic brain. Antioxid Redox Signal. 2015;22(2):149-160.

38. Devonshire IM, Mayhew JE, Overton PG. Cocaine preferentially enhances sensory processing in the upper layers of the primary 
sensory cortex. Neuroscience. 2007;146(2):841-851.

39. Krupinski J, Stroemer P, Slevin M, Marti E, Kumar P, Rubio F. Three-dimensional structure and survival of newly formed blood vessels after focal cerebral ischemia. Neuroreport. 2003;14(8):1171-1176.

40. Kusaka N, et al. Enhanced brain angiogenesis in chronic cerebral hypoperfusion after administration of plasmid human vascular endothelial growth factor in combination with indirect vasoreconstructive surgery. J Neurosurg. 2005;103(5):882-890.

41. Marti HH. Angiogenesis--a self-adapting principle in hypoxia. EXS. 2005;(94):163-80.

42. Morrell JI, Basso JC, Pereira M. Both high and low doses of cocaine derail normal maternal caregiving - lessons from the laboratory rat. Front Psychiatry. 2011;2:30.

43. Boylan JF, et al. Cocaine toxicity and isoflurane anesthesia: hemodynamic, myocardial metabolic, and regional blood flow effects in swine. J Cardiothorac Vasc Anesth. 1996;10(6):772-777. 